\title{
Enhanced 2D plotting method for scanning probe microscopy imaging
}

\author{
G.V. Beketov \\ V. Lashkaryov Institute of Semiconductor Physics, NAS of Ukraine \\ 41, prospect Nauky, 03028 Kyiv, Ukraine; e-mail: gbeketov@isp.kiev.ua
}

\begin{abstract}
An enhanced 2D plotting method for scanning probe microscopy imaging implementing a gradient-based value mapping for pseudocolor images and its application to studies of epitaxial layer surface morphology is presented. It is demonstrated that this method is capable of revealing the finest features on growth surfaces. Presence of elementary growth steps on the surface of flat-topped hillocks found on $\mathrm{Hg}_{0.8} \mathrm{Cd}_{0.2} \mathrm{Te}$ LPE-grown epitaxial layers, examples of cooperative effects of screw dislocations on $\mathrm{PbTe}$ and $\mathrm{Hg}_{1-\mathrm{x}} \mathrm{Cd}_{\mathrm{x}} \mathrm{Te}$ epilayer growth as well as atypical surface morphology of PbTe epilayers are discussed.
\end{abstract}

Keywords: scanning probe microscopy, pseudocolor mapping, epitaxial layer, elementary growth mechanisms, growth steps, dislocations.

Manuscript received 03.02.10; accepted for publication 02.12.10; published online 28.02.11.

\section{Introduction}

As research activities in many natural sciences are becoming increasingly focused on nanoscale objects, the necessity of high resolution imaging instruments is permanently growing. Introduction of scanning probe microscopy (SPM) followed after the invention of scanning tunneling microscopy (STM) by Binnig and Rohrer [1] have revolutionized the techniques of nanoscale imaging. Nowadays, its principles are well established and described in detail [2-5]. An ever increasing number of different SPM models from relatively simple and inexpensive devices to powerful multi-purpose instruments is becoming commercially available. This enables their use in a wider range of applications and on a regular basis, though it also has its underside. Until now there is no generally accepted standard for the SPM datafile format, which hampers data exchange, comparison of results obtained using different models, and usage of the third party software for SPM data analysis. The problem has been recognized by the SPM community, and several research groups as well as private companies took steps to bridge this gap by introducing the SPM freeware that is able to read most of the SPM file formats $[5,6]$. Worthy of mention is the fact that development of one of these program packages, Gwiddion, is currently funded by Czech Metrology Institute.

Russia was the first among former Soviet Republics to proceed to development of SPM instruments and software. At present, Russia is presented on the market of SPM equipment by two companies, two of them, NT-MDT (Zelenograd) and Advanced Technologies Center (Moscow), operating already over twenty years. Their robust and relatively inexpensive scanning probe microscopes are the most affordable instruments for research centers in CIS countries. Unfortunately, the file format introduced by the Advanced Technologies Center for FemtoScan ${ }^{\mathrm{TM}}$ scanning probe microscope was until now unreadable for any SPM processing software available in Ukraine. Since this device has been developed predominantly for life science applications, the original software that comes with it misses certain features important for material sciences. This motivated us to develop the FemtoScan Data Converter (FSDC), a software that can convert FemtoScan data files to more popular formats and incorporates tools for tasks specific to surface science.

In this paper, we describe relevant features of FSDC software and give examples of its application to studies of epitaxial layer surface morphology. 


\section{Overview of relevant features of FSDC software}

The FSDC program has been primarily designed as a supplement to the standard data processing package supplied with the FemtoScan SPM for extending its capabilities and providing access to additional treatment routines implemented in third party software. Its output file is currently compatible with the NanoScope IIITM file format ver. 0x0440000A that can be recognized by most of the SPM data processors. In addition, FSDC itself incorporates several basic procedures intended mainly for imaging and characterization of crystal growth surfaces and is capable of recognizing several other most common data file formats. Typically, growth surface relief represents relatively flat treads with elementary growth steps and terraces resulted from step bunching with heights ranging from only few tenths to few nanometers [4]. This small range of heights complicates obtaining good quality images in several ways. First, special measures should be taken to achieve sufficient contrast, second, precise levelling the surface is required, and, finally, an adverse effect of noise should be suppressed. Virtually all SPM image processors can handle these problems in many different ways. For example, the WSxM software, among other processes for high frequency noise removal, provides Gaussian smooth, which involves averaging image points with a Gaussian weight [5]. We have supplied the FSDC program with several tools, discussed below, which, to our experience, are the most essential for intensive study of such objects.

\subsection{Enhanced 2D plotting method}

Basic two-dimensional data visualization method is the pseudocolor mapping. A pseudocolor image is derived from a set of raw data values by mapping each pixel value to a color according to a table or using analytical expressions. In most of the SPM image processors raw data values are mapped to colors linearly or using an editable broken line (Fig. 1). The latter method enables the user to optimise mapping results for higher in depth resolution and better appearance of the image. However, this approach is quite laborious, which should be considered as its major disadvantage. Moreover, reliable vizualization of growth features requires additional techniques, because deviations of elementary growth step and terrace treads from flatness are comparable with the height of step edges. To facilitate generation of high quality pseudocolor map, current version of Gwiddion image processor provides four different 2D plotting methods. For example, in the adaptive data mapping, the full data range corresponds to the full color range, however data values are mapped to colors non-linearly. The mapping function is based on inverse cumulative height distribution, therefore flat areas generally get augmented interval of the color gradient [6]. Due to this nonlinearity, smaller value variations can be better discerned on them as compared with the regular linear mapping.

We developed enhanced 2D plotting method implementing a gradient-based value mapping for pseudocolor images, similar to a technique recently proposed by A. Visvanathan et al. [7]. In our method, generic data-to-color transforms are given by the following expressions:

$$
\begin{aligned}
& B(R G B)= \\
& =256 \cdot\left\{\frac{\operatorname{arctg}\left[-2 \cdot\left(z-z_{0}(R G B)\right) \cdot C \cdot C_{1}(R G B)\right]}{{ }_{i} P}+127\right\},
\end{aligned}
$$

$$
B(R G B)=256 \cdot \exp \left\{-\left[z-z_{0}(R G B)\right]^{2} \cdot C \cdot C(R G B)\right\}
$$

where $B(R G B)$ is brightness of red, green, and blue components of color palette, $z$ is a raw data value normalized to unity, $z_{0}(R G B)-$ color dependent central point of the curve, $C$ and $C_{1}(R G B)$ - common and colordependent contrast factors, respectively. They specify two different sets of curves presented in Fig. 1a and 1b. Adjustable parameters $z_{0}(R G B), C$, and $C_{1}(R G B)$ allow for the image brightness, contrast, hue, and saturation control.

Expression (1) gives the basic transform to recast the 32-bit raw data values into the 24-bit pseudocolor space. It approaches the ends of the 8-bit intervals for different colors asymptotically, thus preventing

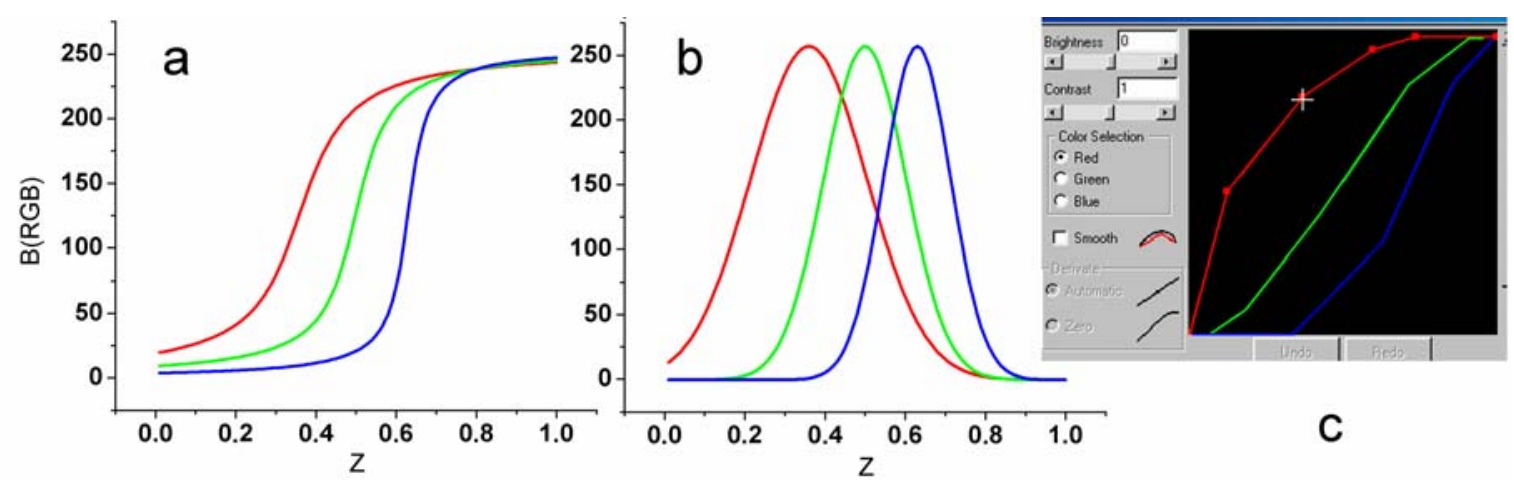

Fig.1. Generic data-to-color transforms (a, b); color setting window of WSxM SPM image processor (c). 
occurrence of "blind" areas when mapping extended features, running in $z$-direction, like hillocks, crevices, and pits. For better visualization of the interior of this type features, an arbitrary number of additional transforms given by expression (2) can be applied.

Further enhancement of imaging of low-height structures, like monoatomic steps and step bunches, is attained with the gradient-based value mapping. Gradient components are calculated using standard numerical procedures for each point of the data array and stored in a separate array of the same size. Then full gradient values (positive or negative) can be added to color intensity values, producing visual effect of 3D images of step edges. Alternatively, they can be used to enhance contrast of the 2D image. For this purpose, small gradient values are to be filtered out and the surface should be restored by integration. The resulting values should then be added to the raw data when producing the $2 \mathrm{D}$ image. Effect of this procedure is shown in Fig. 2. The sample is a $\mathrm{Hg}_{0.8} \mathrm{Cd}_{0.2}$ Te liquid phase epitaxial layer on a $\mathrm{Cd}_{1-\mathrm{y}} \mathrm{Zn}_{\mathrm{y}} \mathrm{Te}$ substrate $(y=0.04)$. A pit-like defect surrounded by terraces is viewed. Comparison of a regular 2D mapping (Fig. 2c) with the image produced using a procedure described above (Fig. 2a) clearly demonstrates drastic improvement in the in-depth resolution. In fact, this approach is equivalent to a precise levelling and straightening of the surface and application of a high contrast data mapping transform. An important point is that this method affects only the pseudocolor map, not influencing the raw data. To improve profiling of small height features, other techniques were implemented.

\subsection{Averaging-enhanced profiling and surface levelling}

One of the most important advantages of SPM is the capability of extracting the surface profile and performing direct measurement of its features. Program tools for profile analysis are integral parts of any SPM data processor. For crystal surfaces, measurement of step heights provides important information about elementary growth mechanisms. However, nonuniformity and time instability of the surface-probe interaction, acoustic vibrations, and the electronic circuitry noise can noticeably compromise the precision of the surface profiling, especially in the case of low-height features.

Most common techniques for noise reduction rely upon data averaging. Nevertheless, averaging across the whole image area will also blur the steps themselves. In FSDC, the averaging-enhanced profiling uses data averaging along segments parallel to the step, thus preventing step blurring. This technique is similar to that implemented in the Digital Instruments Nano Scope software and supplemented with the user-controlled manual plaifitting tool. The example of the growth steps profile shown in Fig. 3 illustrates the application of this

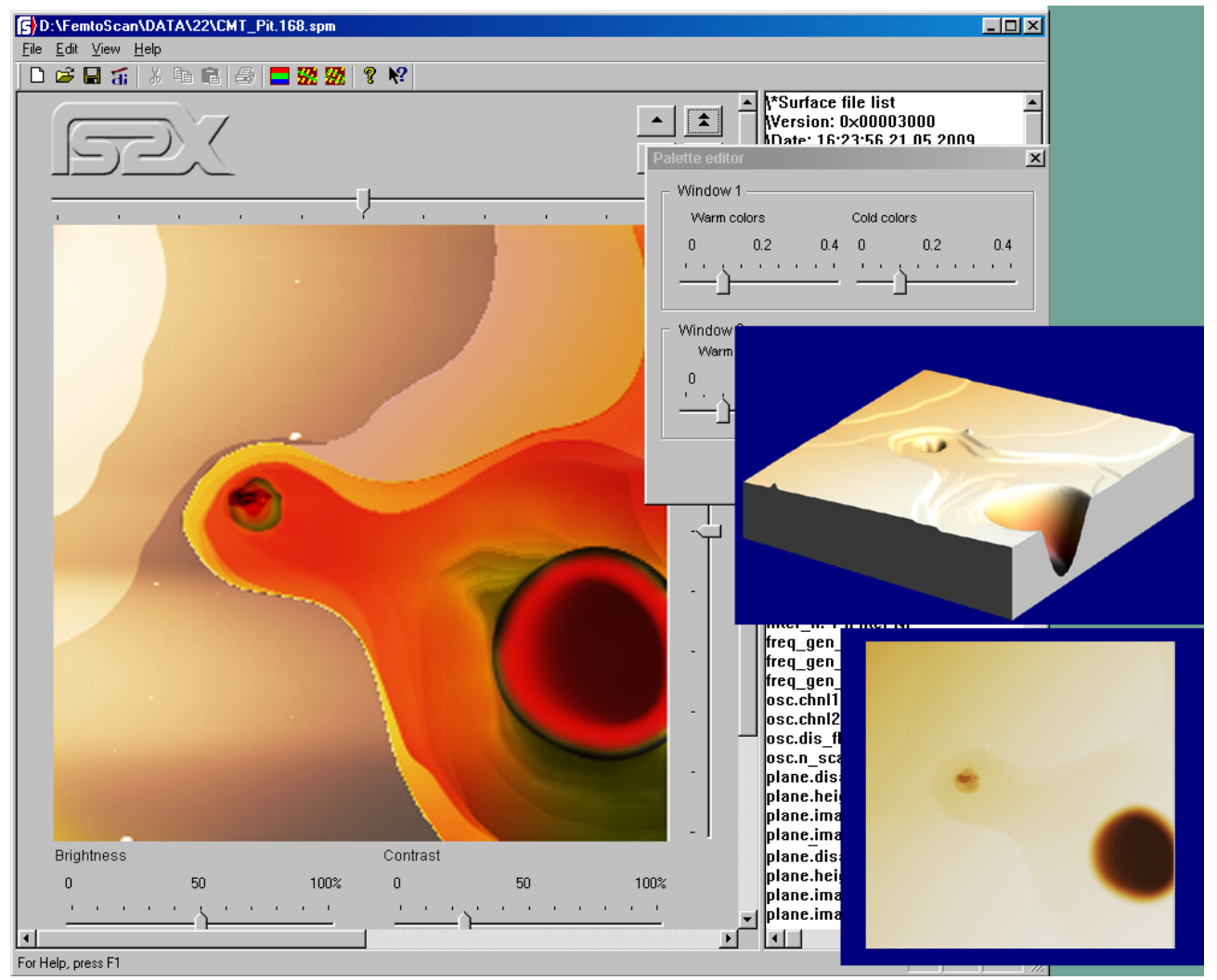

Fig.2. Main window of FSDC program with enhanced 2D plot of the $\mathrm{Hg}_{0.8} \mathrm{Cd}_{0.2} \mathrm{Te} \mathrm{LPE}$ epitaxial layer including a pit-like defect (a); 3-dimensional image of this sample (b); 2-dimensional image without enhancement (WSxM). 


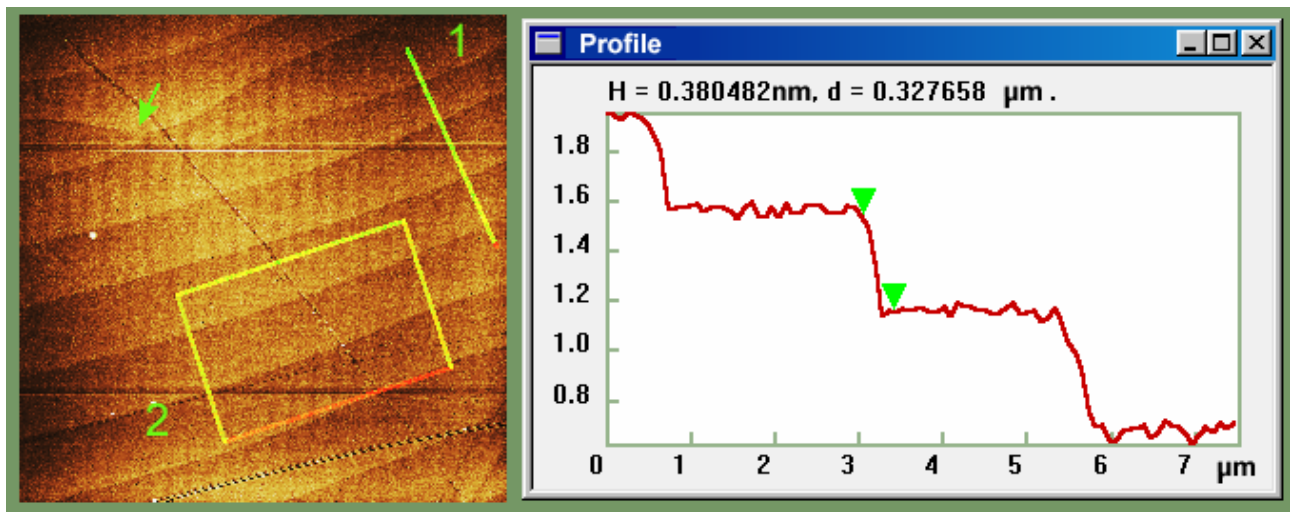

Fig. 3. 2D plot of the $\mathrm{Hg}_{0.8} \mathrm{Cd}_{0.2} \mathrm{Te}$ LPE epitaxial layer with monoatomic growth steps and the averaged profile of the area contained within the box 2 .

approach. An important point is that step treads look horizontal in this plot, that was achieved due to appropriate surface levelling using the interactive plaifitting tool. Otherwise, the saw-like profile would be generally obtained, thus complicating precise measurement of the step height because of its finite width (Fig. 4).

\section{Examples of epitaxial layer surface morphology visualization by using FSDC}

Continuous crystal growth at low supersaturations had been first explained by Burton, Cabrera, and Frank [8]. They showed how the emergence points of dislocations with screw component can act as a permanent generator of surface ledges and derived equations that describe the rate of advance of monomolecular steps. In particular, this theory for crystal growth on stepped surfaces, known as BCF, predicts that in most cases of growth from the vapour the rate of advance of steps will be independent of their crystallographic orientation, so that a growing closed step will be circular. They also analysed cases in which several or many dislocations are involved and showed that they would commonly differ little from the case of a single dislocation.

Part of SPM images presented below illustrate the basic concepts of the BCF theory, while the others possibly provide examples of different growth mechanisms.

\subsection{Flat-topped hillocks}

It is apparent that crystal growth assisted by screw dislocations would result in formation of hillocks on the growth surface. According to the BCF surface diffusion model, hillock shape depends on two characteristic distances: the mean kink distance on the elementary step, $x_{0}$, and the diffusion distance of adatoms on the surface, $\lambda_{s}$. Pyramidal hillocks with well defined facets grow when $\lambda_{s}<x_{0}$. If this condition is not met, poorly polygonized and rounded growth steps are formed [9]. Both types of hillocks are frequently observed on real growth surfaces
[10-12]. Besides, hillocks with flat top are also of frequent occurrence, though it does not follow immediately the BCF theory. To get deeper insight into the mechanism of flat-topped hillock formation, close examination of growth features at their surfaces is required. Regular imaging techniques usually fail to reveal low-height details on protruded structures because of their relatively low contrast. The enhanced 2D plotting method overcomes this difficulty. In Fig. 5, the enhanced 2D image of a flat-topped hillock on a $\mathrm{Hg}_{0.8} \mathrm{Cd}_{0.2}$ Te liquid phase epitaxial layer is shown. For comparison, 2D plot and 3D image built using Gwiddion SPM data processor are presented. The detailes on the epitaxial layer growth conditions were outlined earlier [13].

The enhanced 2D plotting of the hillock top surface clearly reveals elementary growth steps (Fig. 5a), which is an indicator of the layer-by-layer growth mode. It is interesting, however, that the advance of monomolecular steps ceases when they reach the flank of the hillock (arrow 2 in Fig. 5a). The nature of this behaviour is still being debated. Formally, the flat-topped hillock can be considered just as a giant step bunch. By a step bunch it is meant an agglomeration of elementary steps approximately parallel to one another. The step bunching induced by surface diffusion is relatively well understood from studies of the vapour growth or MBE of simple substances [14]. Between parallel steps, the surface diffusion field is one-dimensional, so that the concentration of the crystallizing species in the adsorbed layer changes exponentionally with the distance from the step. This results in a lower material supply to closer steps and retardation of their propagation rate as compared with that of widely separated steps.

Finally, this nonlinear interaction between growth steps mediated by the diffusion field brings about the formation of step bunches. Adsorption of foreign species at the interstep terraces can also contribute to this nonlinearity. Following BCF, adsorption on interstep terraces and surface diffusion is involved even in solution growth, so that the above mechanism is applicable for explanation of the occurrence of step bunches in LPE as well. 


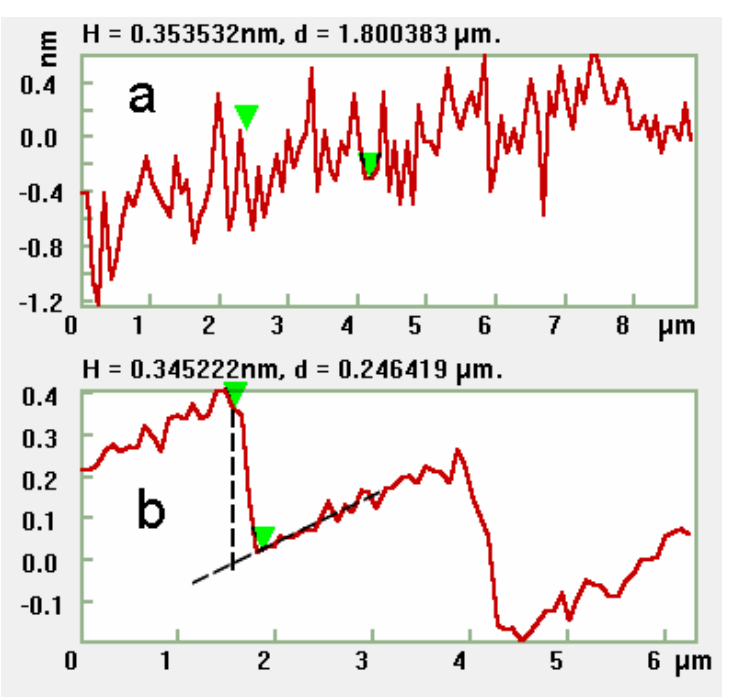

Fig. 4. a) Example of profile concealment by noise if averaging-enhancement is not applied; b) saw-like profile obtained if the image is not levelled complicates precise measurement of the step height.

However, typical step bunching process results in formation of multiple macrosteps $[9,14]$, while there is no sign of terracing on the hillock flanks. The hillock shape implies that its flanks are composed of high-index surfaces. Since they are microscopically rough, their growth may occur by the direct incorporation mechanism. It means that two different growth modes coexist under the same growth conditions. Formation of the macroscopically flat facets was discussed in [15]. If performed under sufficiently low supersaturation, the growth leads to the formation of the face morphology corresponding to the minimum of the surface-free energy. The thermodynamic driving force for face flattening is the difference in the surface-free energy of the vicinal faces of the hillocks, emanating from screw dislocations. The hillock slope gives the quantitative relation between energetics and kinetics. The result of the considerations is that the lower the supersaturation, the more important is the role of the surface-free energy in face flattening.

\subsection{Cooperative dislocations and possible mechanism of crystallinity improvement in the epitaxial growth process}

When the separation between the screw dislocations is small enough, they act conjointly as a combined source of the elementary growth steps. The dislocations with the Burgers vectors of the same sign result in formation of two- or sometimes even three-fold growth spirals, while the dislocations with the Burgers vectors of the opposite sign give rise to islands on the growth surface $[8,9]$.

In Fig. 6, the surface morphology of the $\mathrm{PbTe}$ epitaxial layer on the $\mathrm{BaF}_{2}$ substrate with (111) orientation is shown. The layer was grown using the hot wall technique. Monocrystalline $p$ - $\mathrm{PbTe}$ at temperatures between $300-400{ }^{\circ} \mathrm{C}$ served as a vapour source. The substrate temperature was kept 40 to $60^{\circ} \mathrm{C}$ below the source temperature. Layer thickness was $2-2.5 \mu \mathrm{m}$. More details on the layer growth conditions and its structural and electrical properties can be found in [16]. Two hillocks in Fig. 6 exemplify two cases of elementary growth steps formation by cooperative dislocations, while for the third hillock (on the right top) separation between two dislocations is too large to produce a well-defined cooperative effect. Two nested spiral growth steps were formed instead.

Other characteristic features of surface morphology are numerous pits ranging approximately from 50 to $150 \mathrm{~nm}$ in diameter and from 15 to $120 \mathrm{~nm}$ in depth. Similar pits were also observed on other epitaxial layers, grown from gaseous phase, for instance, on $\mathrm{AlGaN} / \mathrm{GaN}$ epitaxial layer grown on a Si substrate [17]. In this work, pit arrays forming a network structure were observed by an atomic force microscopy (AFM). Investigation using a transmission electron microscopy confirmed that pit arrays represent surface termination of edge dislocations at the small-angle boundaries of slightly misoriented crystal domains in the substrate. They are formed because the elastic strain in the vicinity of the
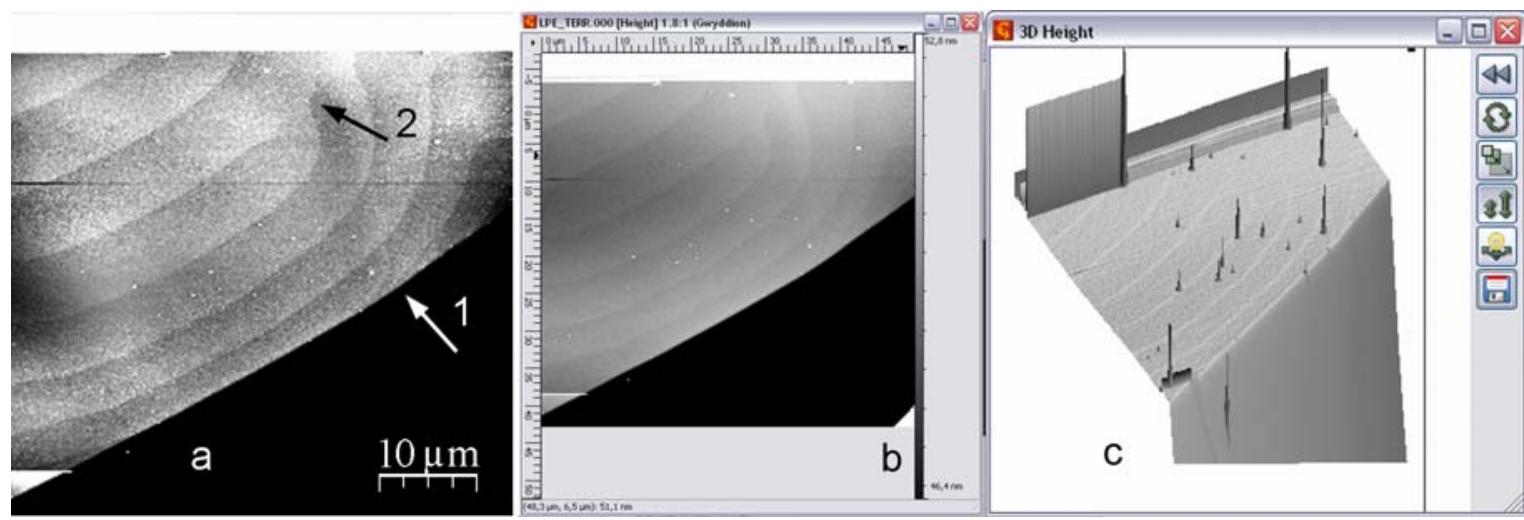

Fig. 5. a) enhanced 2D plot of flat-topped hillock revealing elementary growth steps; b) the same hillock viewed by WSxM SPM data processor; c) 3D image of this hillock built using Gwiddion software.

(C) 2011, V. Lashkaryov Institute of Semiconductor Physics, National Academy of Sciences of Ukraine 
dislocation core result in local increase of the Gibbs free energy and lower uptake probability of crystallizing species. Apparently, pits in Fig. 6 are of the same origin.

For industrial applications, substrates oriented slightly off the precise (111) plane are normally used. This misorientation guarantees the source of elementary growth steps required for a layer-by-layer growth mode and result in superior crystallinity of the epitaxial layers [18]. Though screw dislocations evidently exist in the substrates, which are usually slices cut from single crystals [19], they rarely pierce the epitaxial layer. While "healing" the misfit dislocations is widely covered in the literature, the behaviour of screw dislocations during epitaxial growth on misoriented substrates received less attention.

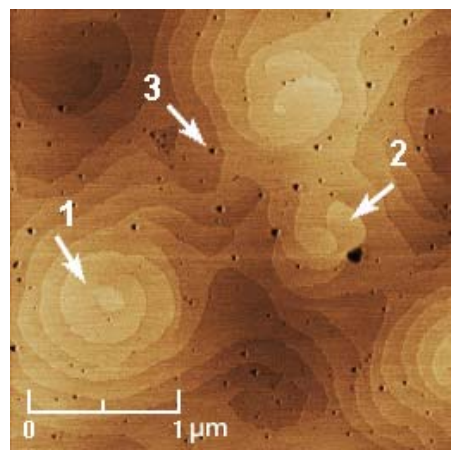

Fig. 6. Growth surface of $\mathrm{PbTe}$ epitaxial layer on $\mathrm{BaF}_{2}$ substrate. 1 - elementary island on the top of the hillock formed due to a cooperative effect of screw dislocations with the Burgers vectors of the same sign; 2 - double spiral originating from cooperative screw dislocations with the Burgers vectors of the opposite sign; 3 - pit probably formed in the course of surface termination of edge dislocation.
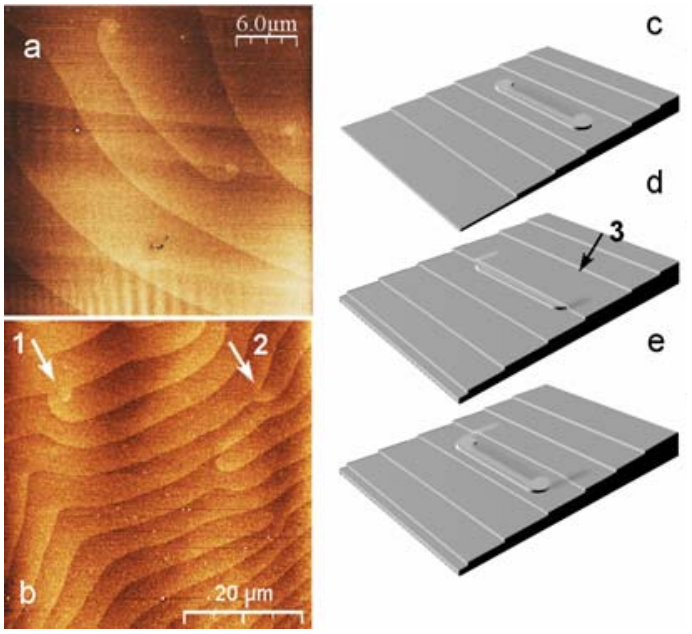

Fig. 7. Surface morphology of $\mathrm{Hg}_{0.8} \mathrm{Cd}_{0.2} \mathrm{Te}$ LPE-grown epitaxial layers on $\mathrm{Cd}_{1-\mathrm{y}} \mathrm{Zn}_{\mathrm{y}} \mathrm{Te}(\mathrm{y}=0.04)$ substrates with orientation (111) $\pm 0.5^{\circ}$. (a) Local growth step resulting from the cooperative effect of screw dislocations with Burgers vectors of opposite signs; (b) interaction of local growth steps with the flux of advancing steps formed due to substrate misorientation; (c, d, e) a model of "sweeping out" screw dislocations. The arrow 3 points at a buried edge dislocation.
Examination of surface features presented in Fig. 7 may shed light on the mechanism of "sweeping out" screw dislocations by the flux of elementary growth steps. The samples are $\mathrm{Hg}_{0.8} \mathrm{Cd}_{0.2} \mathrm{Te} \mathrm{LPE}$-grown epitaxial layers on $\mathrm{Cd}_{1-\mathrm{y}} \mathrm{Zn}_{\mathrm{y}} \mathrm{Te}(\mathrm{y}=0.96)$ substrates with orientation $(111) \pm 0.5^{\circ}$. Growth procedure was similar to that employed in [13]. In Fig. 7, a growth step terminated by two screw dislocations with Burgers vectors of opposite signs is clearly seen. In Fig. $7 \mathrm{~b}$ the arrow 1 points at an identic feature partially overlapped by the next elementary step. Feature pointed out by the arrow 2 most likely represents one of analogous dislocation-induced steps with completely buried spiral origins. These observations suggest the appearance model of gradual displacement of screw dislocation cores by sequence of advancing elementary growth steps following by generation of edge dislocations parallel to the surface (Fig. 7c,d,e). The latter image in this figure depicts hypothetic strike-through of displaced screw dislocations. Finally, this process should result in transformation of purely screw dislocation in the substrate into a mixed dislocation consisting of both screw and edge sections with the averaged dislocation line approximately parallel to the surface. To confirm this hypothesis, experimental studies using transmission electron microscopy are required.

\subsection{Evidences for contribution of vapour-liquid-solid growth mechanism to vapour phase epitaxy}

Edges of the considered above growth steps had smooth contours. Not frequently encountered on epilayer surfaces are also elementary steps of irregular shape. An example of PbTe epitaxial layer with peculiar surface morphology is presented in Fig. 8. Like to the layer shown in Fig. 6, it was grown on $\mathrm{BaF}_{2}$ substrate by hot wall technique under similar conditions, but using another apparatus [20]. Ledges of growth steps on its surface have distinctive protrusions reminiscent of the tour d'Eiffel silhouette with spherical particles $50-70 \mathrm{~nm}$ in diameter at their points. When AFM operates in the contact mode, some of these spheres usually disappear after several frames have been recorded. Characteristic flutter of the cantilever at this moment suggests that the spheres are removed by the probe tip.

These observations may lead to a conclusion that spheres represent metal droplets. Evidences for metal droplets formation during vapour-phase crystallization are abundant in the literature [21-23]. The droplets may hasten the advance of elementary steps due to additional contribution of the vapour-liquid-solid (VLS) growth mechanism. This mechanism is a wellestablished phenomenon [24] and enables growth of three-dimensional filiform crystals and nanowires [25]. On the other hand, the results presented here show that lateral nanowire growth on a suitable substrate is possible as well. 


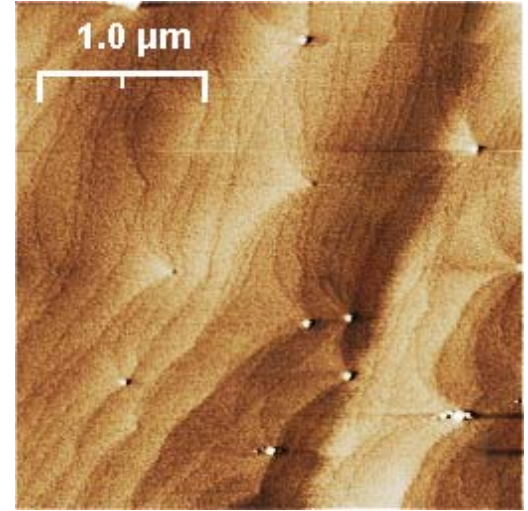

Fig. 8. "Atypical" surface morphology of PbTe epitaxial layer. Spherical particles at points of growth step protrusions possibly represent metal droplets.

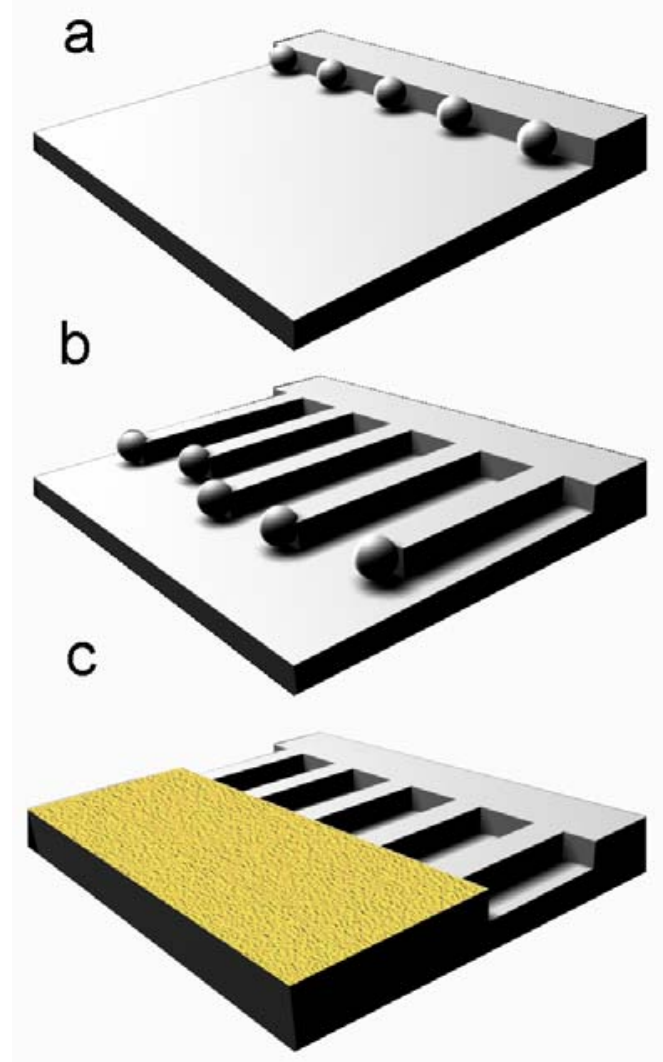

Fig. 9. A model of prostrate nanowire growth on a substrate by the VLS mechanism. a) initial step with deposited metallic nanoparticles, b) nanowire growth, c) the resulting nanowire array with the metal contact.

Let us consider a thought model shown in Fig. 9. Suppose that the epitaxial layer grown on a suitable substrate, to say, Si on sapphire, is partially removed to form a step (Fig. 9a), and metal nanoparticles are deposited at this step (formation of Si filiform crystals is usually performed using $\mathrm{Au}$ droplets). It seems conceivable that, under appropriate supersaturation, the
VLS mechanism would result in a lateral nanowire growth, as shown in Fig. 9b. Possibility of isolated monocrystalline hillock formation has been already proved experimentally [26]. Finally, a metal layer could be deposited to provide an electrical contact to the nanowire array. Structures of this type are now extensively studied in view of evolution of novel electronic devices like single electron transistors (SETs), considered candidates as elements for future low power, high density integrated circuits [27].

\section{Conclusions}

An enhanced 2D plotting method for scanning probe microscopy imaging was developed and implemented in FSDC software for conversion of FemtoScan SPM data files to other formats. Relevant features of FSDC software and examples of their application to studies of epitaxial layer surface morphology are described.

Presence of elementary growth steps on the surface of flat-topped hillocks found on $\mathrm{Hg}_{0.8} \mathrm{Cd}_{0.2} \mathrm{Te}$ LPEgrown epitaxial layers was demonstrated. Examples of cooperative effects of screw dislocations on $\mathrm{PbTe}$ and $\mathrm{Hg}_{1-\mathrm{x}} \mathrm{Cd}_{\mathrm{x}} \mathrm{Te}$ epilayer growth was considered, and a model of "sweeping out" screw dislocations by the flux of elementary growth steps was proposed.

The example of $\mathrm{PbTe}$ epitaxial layer with atypical surface morphology was discussed as a possible evidence for contribution of vapour-liquid-solid growth mechanism to vapour phase epitaxy. A thought model for nanowire array formation method based on this effect has been proposed.

\section{References}

1. G. Binnig, H. Rohrer, Ch. Gerber, and E. Weibel, Surface studies by scanning tunneling microscopy // Phys. Rev. Lett. 49(1), p. 57-61 (1982).

2. Procedures in Scanning Probe Microscopies, R.J. Colton, A. Engel, J.E. Frommer, H.E. Gaub, A.A. Gewith, R. Guckenberger, J. Rabe, W.M. Heckl, B. Parkinson (editors). John Wiley \& Sons, Chichester, 1998.

3. R. Wiesendanger, Scanning Probe Microscopy and Spectroscopy: Methods and Applications. Cambridge University Press, Cambridge, 1994.

4. R. Howland, L. Benatar, A Practical Guide to Scanning Probe Microscopy, Park Scientific Instruments, Sunnyvale, CA, 1993-1997.

5. I. Horcas, R. Fernández, J.M. Gómez-Rodríguez, I. Horcas， R. Fernández， J. Colchero， J. GómezHerrero, A.M. Baro, WSXM: A software for scanning probe microscopy and a tool for nanotechnology // Rev. Sci. Instrum. 78, p. $013705-$ 1 - 013705-8 (2007).

6. P. Klapetek, D. Necas, C. Anderson, Gwiddion User Guide. Czech Metrology Institute, 2004-2007, 2009. 
7. A. Visvanathan, S.E. Reichenbach, Q. Tao, Gradient-based value mapping for pseudocolor images // J. Electron. Imaging 16(3), p. 033004-1 033004-8 (2007).

8. W.K. Burton, N. Cabrera, F.C. Frank, The growth of crystals and the equilibrium structure of their surfaces // Phil. Trans. Roy. Soc. 243(866), p. 299358 (1951).

9. K. Sangwal, Growth kinetics and surface morphology of crystals grown from solutions: recent observations and their interpretations // Progr. Crystal Growth and Charact. 36(3), p. 163248 (1998).

10. P.R. Hageman, V. Kirilyuk, W.H.M. Corbeek, J.L. Weyher, B. Lucznik, M. Bockowski, S. Porowski, S. Muller, Thick GaN layers grown by hydride vapor-phase epitaxy: hetero- versus homo-epitaxy// J. Cryst. Growth 255, p. 241-249 (2003).

11. A. Tallaire, M. Kasu, K. Ueda and T. Makimoto, Origin of growth defects in CVD diamond epitaxial films // Diamond and Related Materials 17(3), p. 60-65 (2008).

12. Y.K. Kim, J.Y. Lee, H.S. Kim, J.-H. Song, S.-H. Suh, An electron microscopy study on the formation mechanism of hillocks on the (100)CdTe/GaAs // J. Cryst. Growth 192(1-2), p. 109-116 (1998).

13. G.V. Beketov, L.V. Rashkovetskiy, O.V. Rengevych, G.I. Zhovnir, AFM study of micromorphology and microscopic growth mechanisms of $\mathrm{Hg}_{1-\mathrm{x}} \mathrm{Cd}_{\mathrm{x}} \mathrm{Te} \mathrm{LPE}$ epitaxial layers // Semiconductor Physics, Quantum Electronics \& Optoelectronics 3(1), p. 45-51 (2000).

14. A.A. Chernov, Notes on interface growth kinetics 50 years after Burton, Cabrera and Frank, In: 50 Years Progress in Crystal Growth. A reprint collection. R.S. Feigelson (editor). Elsevier, Amsterdam, p. 47-66 (2004).

15. C.N. Nanev, Is crystal growth under low supersaturations influenced by a tendency to a minimum of the surface-free energy? // Annals of the New York Academy of Sciences 1077, p. 194207 (2006).

16. G.V. Beketov, S.P. Movchan, F.F. Sizov, Layerby-layer growth of $\mathrm{PbTe}$ epitaxial layers and properties of heterojunctions formed on their basis // Optoelektronika i poluprovodnikovaya tehnika 35, p. 135-145 (2000), in Russian.
17. S. Hitoshi, K. Sadahiro, M. Takeyoshi, S. Yoshihiro, I. Masayuki, Y. Seikoh, Investigation of surface defect structure originating in dislocations in $\mathrm{AlGaN} / \mathrm{GaN}$ epitaxial layer grown on a Si substrate // J. Cryst. Growth 298, p. 305309 (2007).

18. L.A. Almeida, M. Groenert, J. Markunas, J.H. Dinan, Influence of substrate orientation on the growth of $\mathrm{HgCdTe}$ by molecular beam epitaxy // $\mathrm{J}$. Electron. Mater. 35(6), p. 1214-1218 (2006).

19. Bulk Crystal Growth of Electronic, Optical \& Optoelectronic Materials, P. Capper (editor). John Wiley \& Sons, Chichester, 2005.

20. G.V. Lashkarev, M.V. Radchenko, E.I. Slynko, V.N. Vodopianov, G.V. Beketov, E.V. Rengevich, Hot wall growth and properties of lead telluride films doped by germanium and gallium // Semiconductor Physics, Quantum Electronics \& Optoelectronics 3(3), p. 295-299 (2000).

21. V.P. Chaly, B.A. Borisov, D.M. Demidov, D.M. Krasovitsky, Yu.V. Pogorelsky, A.P. Shkurko, I.A. Sokolov, S.Yu. Karpov, Indium droplet formation during molecular beam epitaxy of InGaN // J. Cryst. Growth 206, p. 147-149 (1999).

22. Jeong-Rae Ro, Sung-Bock Kim, El-Hang Lee, Formation of $\mathrm{GaAs}$ island by Ga-droplet-induced chemical beam epitaxy // J. Cryst. Growth 188, p. 377-382 (1998).

23. P.R. Hageman, V. Kirilyuk, W.H.M. Corbeek, J.L. Weyher, B. Lucznik, M. Bockowski, S. Porowski, S. Muller, Thick GaN layers grown by hydride vapor-phase epitaxy: hetero-versus homoepitaxy // J. Cryst. Growth 255, p. 241-249 (2003).

24. E.I. Givargizov, Growth of Filiform and Lamellar Crystals from Vapour. Nauka, Moscow, 1977 (in Russian).

25. S.K. Chan, N. Liu, Y. Cai, N. Wang, G.K.L. Wong, I.K. Sou, Molecular beam epitaxy - grown $\mathrm{ZnSe}$ nanowires // J. Electron. Mater. 35(6), p. 12461250 (2006).

26. Zh. Yan, Sh. Naritsuka, T. Nishinaga, Interface supersaturation dependence of step velocity in liquid-phase epitaxy of InP // J. Cryst. Growth 198/199, p. 1077-1081 (1999).

27. U. Hashim, A. Rasmi, Single-electron transistor (SET) process and device simulation using SYSNOPSYS TCAD tools // Amer. J. Appl. Sci. 3(7), p. 1933-1938 (2006).. 